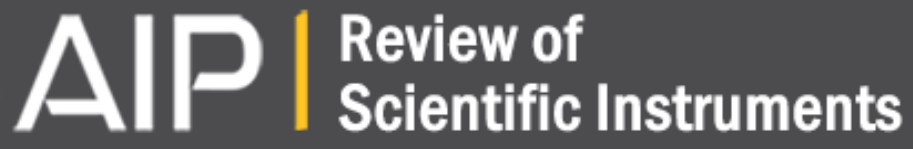

\section{A high-bandwidth amplitude estimation technique for dynamic mode atomic force microscopy}

K. S. Karvinen and S. O. R. Moheimani

Citation: Review of Scientific Instruments 85, 023707 (2014); doi: 10.1063/1.4865841

View online: http://dx.doi.org/10.1063/1.4865841

View Table of Contents: http://scitation.aip.org/content/aip/journal/rsi/85/2?ver=pdfcov

Published by the AIP Publishing

\section{Articles you may be interested in}

Microcantilevers with embedded accelerometers for dynamic atomic force microscopy

Appl. Phys. Lett. 104, 083109 (2014); 10.1063/1.4866664

Subharmonic excitation in amplitude modulation atomic force microscopy in the presence of adsorbed water layers

J. Appl. Phys. 110, 114902 (2011); 10.1063/1.3663437

Fast imaging with alternative signal for dynamic atomic force microscopy

Appl. Phys. Lett. 97, 133101 (2010); 10.1063/1.3495987

Theory of phase-modulation atomic force microscopy with constant-oscillation amplitude J. Appl. Phys. 103, 064317 (2008); 10.1063/1.2896450

Probe-surface interaction mapping in amplitude modulation atomic force microscopy by integrating amplitudedistance and amplitude-frequency curves

Appl. Phys. Lett. 91, 023122 (2007); 10.1063/1.2756271

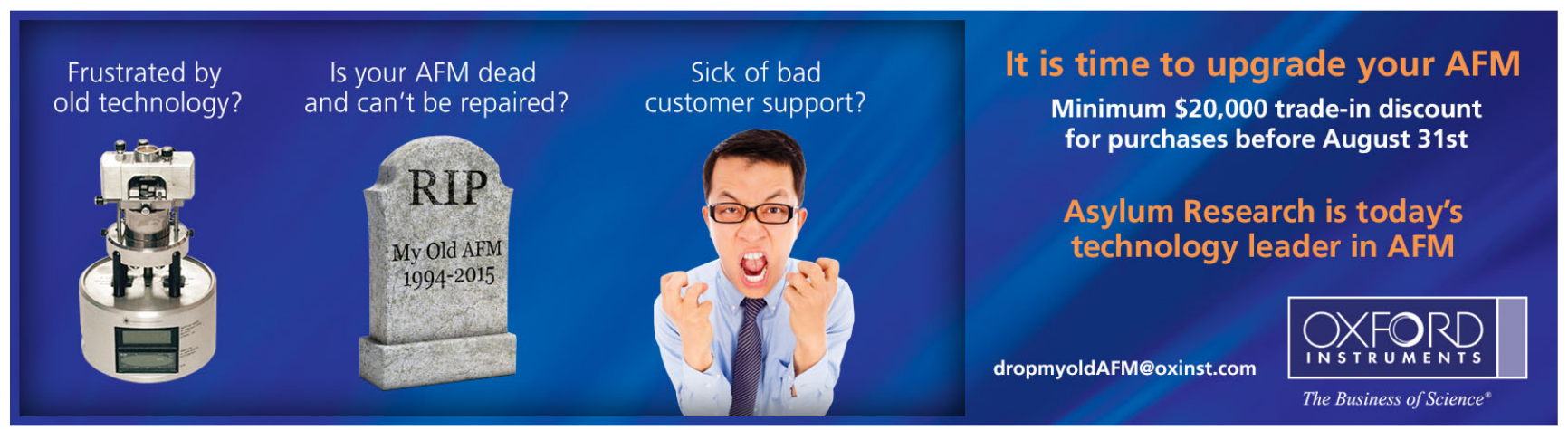




\title{
A high-bandwidth amplitude estimation technique for dynamic mode atomic force microscopy
}

\author{
K. S. Karvinen a) and S. O. R. Moheimanib) \\ School of Electrical Engineering and Computer Science, The University of Newcastle, Callaghan, \\ NSW 2308, Australia
}

(Received 17 November 2013; accepted 1 February 2014; published online 20 February 2014)

\begin{abstract}
While often overlooked, one of the prerequisites for high-speed amplitude modulation atomic force microscopy is a high-bandwidth amplitude estimation technique. Conventional techniques, such as RMS to DC conversion and the lock-in amplifier, have proven useful, but offer limited measurement bandwidth and are not suitable for high-speed imaging. Several groups have developed techniques, but many of these are either difficult to implement or lack robustness. In this contribution, we briefly outline existing amplitude estimation methods and propose a new high-bandwidth estimation technique, inspired by techniques employed in microwave and RF circuit design, which utilizes phase cancellation to significantly improve the performance of the lock-in amplifier. We conclude with the design and implementation of a custom circuit to experimentally demonstrate the improvements and discuss its application in high-speed and multifrequency atomic force microscopy. () 2014 AIP Publishing LLC. [http://dx.doi.org/10.1063/1.4865841]
\end{abstract}

\section{INTRODUCTION}

The invention of the atomic force microscope (AFM) in $1986^{1}$ enabled unprecedented levels of performance in microscopy. Since then, the AFM has been of vital importance in the investigation of the nanoscale.

Of particular significance is amplitude modulation atomic force microscopy ${ }^{2,3}$ (AM-AFM). Despite its suitability for the study of sensitive biological samples, one limitation of AM-AFM is its low imaging bandwidth owing primarily to the dynamics of the microcantilever and the amplitude estimation method. ${ }^{4}$ Microcantilever dynamics play a fundamental role in AM-AFM imaging; they impose a tradeoff between the sensitivity and speed of the measurement. ${ }^{5}$ High $\mathrm{Q}$ factors promote high resolution, operation in the attractive regime, ${ }^{6}$ and low tip-sample force interactions, ${ }^{7}$ suitable for the interrogation of delicate samples but at the expense of the imaging bandwidth. In addition, owing to the reliance on the regulation of the measured amplitude to estimate the sample topography, the bandwidth of the estimation technique clearly limits the performance of AM-AFM techniques. While AM-AFM is generally not associated with high-speed imaging, the development of high-speed AM-AFM is a topic of considerable interest. The observation of dynamic biological processes at the nanoscale is clearly a strong motivation to develop the technology. ${ }^{8-10}$

Conventional amplitude estimation techniques in AFM include RMS to DC conversion and the lock-in amplifier, neither of which are compatible with high-speed imaging. Several research groups have developed alternative highbandwidth amplitude estimation techniques, such as peakhold detection ${ }^{11}$ and the Fourier method. ${ }^{12}$

We review existing amplitude estimation techniques, outlining their advantages and disadvantages. Utilizing the prin-

a)Electronic mail: Kai.Karvinen@uon.edu.au

b) Electronic mail: Reza.Moheimani@newcastle.edu.au ciples of image rejection receivers in RF and microwave applications, we propose a high-bandwidth amplitude estimation technique, which is suitable for use in high-speed AMAFM and multifrequency AFM. We discuss the performance and implications of this method and conclude with experimental verification.

\section{AMPLITUDE ESTIMATION TECHNIQUES IN AFM}

\section{A. RMS to DC conversion}

Possibly the most primitive technique for amplitude estimation is RMS to DC conversion. The RMS value of a periodic signal is defined as

$$
V_{r m s}=\sqrt{\frac{1}{T} \int_{t}^{t+T} V^{2}(t)},
$$

where $T$ is the period of the signal. The rms value of a sinusoidal signal is proportional to the amplitude of the signal: $V_{r m s}=V_{p k} / \sqrt{2}$. In AM-AFM, regulation of the amplitude of oscillation of the driven mode, typically the first flexural mode, is required to estimate the surface topography. However, if other frequency components are present in the signal, such as higher harmonics, RMS to DC conversion cannot accurately determine the oscillation amplitude of the flexural mode.

Methods of true RMS to DC conversion include thermal RMS to DC conversion; direct computation, where amplifiers are used to perform the squaring, averaging, and square rooting functionality; and indirect computation, which utilizes feedback. ${ }^{13}$ A number of analog and digital true RMS to DC converters are commercially available.

\section{B. Mean absolute deviation}

In AM-AFM, "RMS to DC converters" are typically implemented using a rectifier circuit and a low-pass filter. ${ }^{14}$ 

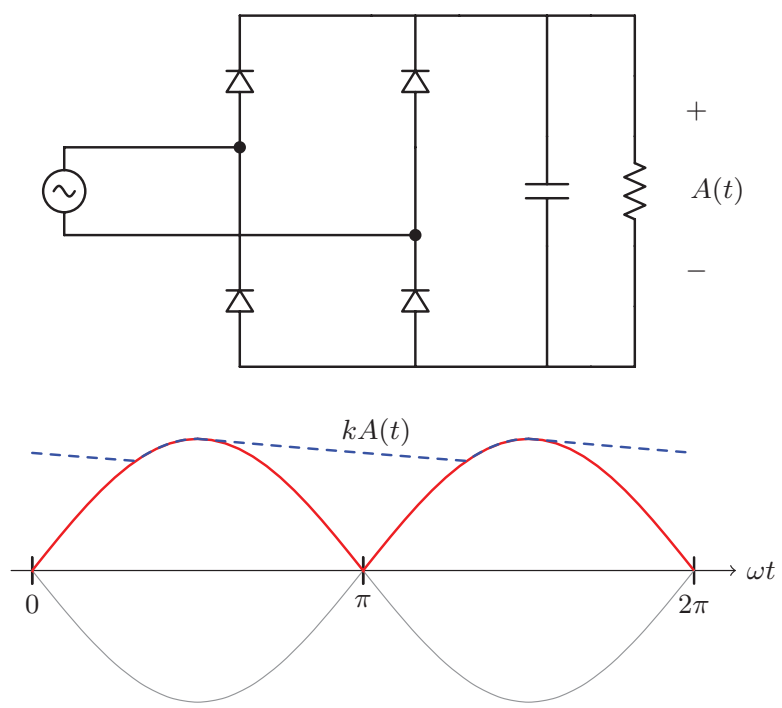

FIG. 1. Mean absolute deviation: amplitude estimation with a full-wave bridge rectifier and low-pass filter.

Such an implementation is highlighted in Fig. 1. However, we must stress that this is not RMS to DC conversion, but mean absolute deviation, ${ }^{13}$ which determines the $\mathrm{AC}$ average of the waveform $2 V_{p k} / \pi$. For a pure sinusoidal signal, the output is clearly proportional to the oscillation amplitude, but this technique suffers from the same drawbacks as RMS to DC conversion. Inaccurate amplitude estimates are obtained when other frequency components are present and high-frequency oscillations must be filtered from the low-bandwidth amplitude estimate. Such techniques often require dozens of cycles for convergence, which severely limits the attainable imaging bandwidth in AM-AFM.

\section{Lock-in amplifier}

In comparison to the aforementioned techniques, the lock-in amplifier is advantageous in that it can extract the amplitude of a specific frequency component. A narrowband detection technique, it is often employed to recover weak signals from noisy measurements. The lock-in amplifier mixes the input signal with quadrature-phased sinusoids and the amplitude information can be extracted from a low-frequency signal. With appropriate filtering, the undesirable frequency content, situated at $2 \omega_{0}$, can be rejected and accurate amplitude estimates can be obtained. However, since low-pass filters must separate the slow time-varying component from high-frequency oscillations, the measurement bandwidth is limited.

In conventional tapping mode AFM, the first flexural mode of the microcantilever is excited. Typically, it is assumed that the higher harmonics are insignificant and the tip displacement is dominated by the first flexural mode. Let us consider

$$
v(t)=V_{0} \cos \left(\omega_{0} t+\phi\right) .
$$
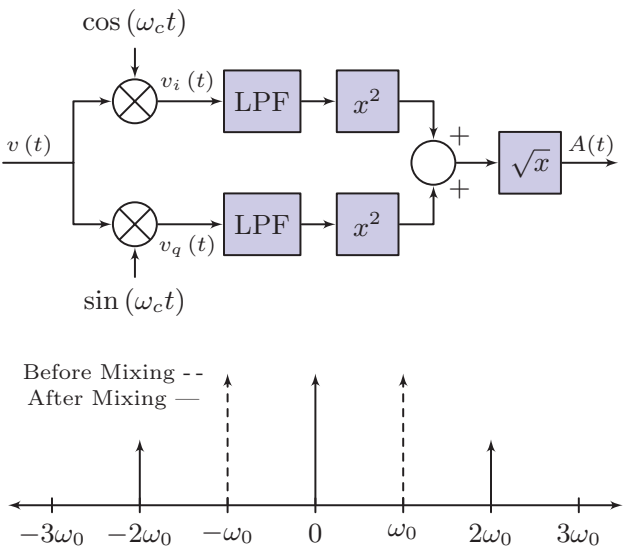

FIG. 2. Lock-in amplifier implementation and operation in the frequency domain when $\omega_{c}=\omega_{0}$.

The in-phase and quadrature outputs are

$$
\begin{aligned}
v_{i}(t) & =V_{0} \cos \left(\omega_{0} t+\phi\right) \cos \left(\omega_{c} t\right) \\
& =\frac{V_{0}}{2}\left[\cos \left(\left(\omega_{0}-\omega_{c}\right) t+\phi\right)+\cos \left(\left(\omega_{0}+\omega_{c}\right) t+\phi\right)\right], \\
v_{q}(t) & =V_{0} \cos \left(\omega_{0} t+\phi\right) \sin \left(\omega_{c} t\right) \\
& =\frac{V_{0}}{2}\left[\sin \left(\left(\omega_{0}+\omega_{c}\right) t+\phi\right)-\sin \left(\left(\omega_{0}-\omega_{c}\right) t+\phi\right)\right] .
\end{aligned}
$$

When $\omega_{c}=\omega_{0}$,

$$
\begin{aligned}
& v_{i}(t)=\frac{V_{0}}{2}\left[\cos \phi+\cos \left(2 \omega_{0} t+\phi\right)\right], \\
& v_{q}(t)=\frac{V_{0}}{2}\left[\sin \left(2 \omega_{0} t+\phi\right)-\sin \phi\right] .
\end{aligned}
$$

Filtering out the high-frequency oscillations, the in-phase and quadrature signals can be combined to determine the amplitude of the displacement signal

$$
\sqrt{\left(V_{0} \cos \phi\right)^{2}+\left(V_{0} \sin \phi\right)^{2}}=V_{0}
$$

Fig. 2 highlights the significance of the frequency components at $2 \omega_{0}$ and emphasizes the limited measurement bandwidth that can be achieved when this component is removed via filtering.

\section{Peak-hold technique}

The peak-hold technique, ${ }^{11}$ developed specifically for high-speed AM-AFM imaging, utilizes sample and hold circuitry to hold both the positive and negative peaks for the duration of the cycle. While this technique is more susceptible to noise as there are only two measurements per cycle, more importantly, the presence of harmonics will result in erroneous amplitude estimates and complicate the synchronization of the sample and hold circuitry. Fig. 3 highlights how the presence of higher harmonics affects the peak-hold technique. 


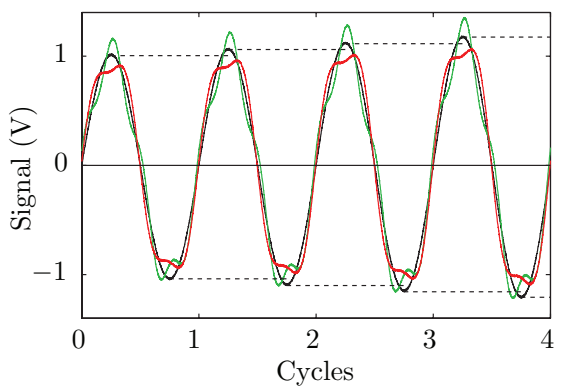

FIG. 3. The peak-hold technique accurately estimates the amplitude of a pure sinusoid $(-)$. Erroneous amplitude estimates result from the presence of small harmonics in addition to the fundamental: $0.15 \sin (3 \omega t+0.2)$ (red dashed line) and $0.15 \sin (4 \omega t+1)$ (green dashed line).

\section{E. Fourier technique}

The Fourier method involves the direct computation of the Fourier series coefficients in one cycle. While this technique offers an improvement over the peak-hold technique, it requires accurate timing and integration over an integral number of oscillations. ${ }^{12}$

The Fourier series coefficients are determined as

$$
\begin{aligned}
& A_{1}(t)=\frac{\omega(t)}{\pi} \int_{0}^{\frac{2 \pi}{\omega(t)}} d(t-\tau) \cos (u(t-\tau)) d \tau, \\
& B_{1}(t)=\frac{\omega(t)}{\pi} \int_{0}^{\frac{2 \pi}{\omega(t)}} d(t-\tau) \sin (u(t-\tau)) d \tau .
\end{aligned}
$$

Performed digitally, the speed and resolution of the analogto-digital converter will affect the accuracy of the calculation. For ultrahigh-frequency microcantilevers, such an implementation could be costly, if not impractical.

\section{F. Image rejection mixer}

Image rejection mixers are commonly employed in microwave and radio frequency systems, particularly in heterodyne receivers. ${ }^{15}$ Engineered specifically to overcome limitations in filter performance and simplify the filter requirements, image rejection receivers utilize phase cancellation techniques to eliminate undesirable mixing products in the output. Since image rejection mixers eliminate frequency components via cancellation and not filtering, they can be used to eliminate closely spaced components of the signal in the frequency domain.

Originating from the single-sideband modulator, the Hartley architecture is considered to be the most basic im- age rejection mixer. ${ }^{15}$ This circuit mixes the input with the quadrature outputs produced by the local oscillator, similar to the lock-in amplifier. Complementary first-order filters create a $90^{\circ}$ phase shift prior to summation, which eliminates the unwanted image in the output. The Hartley architecture is, however, susceptible to circuit mismatches; phase and gain imbalances prevent complete image cancellation. The Weaver architecture introduces a second quadrature mixing operation instead of the RC-CR network. As a result, it offers greater image rejection than the Hartley architecture, but also exhibits sensitivity to circuit mismatches..$^{15}$ These two image-reject architectures are highlighted in Fig. 4.

\section{G. High-bandwidth lock-in amplifier technique}

We have developed a novel high-bandwidth amplitude estimation technique, inspired by image rejection mixers and modulated-demodulated control. ${ }^{16,17}$ The proposed technique is robust to signal noise and accurately determines the amplitude of a specific frequency component while improving the measurement bandwidth.

Our proposed estimation technique is outlined in Fig. 5. By introducing a phase shift of $90^{\circ}$ to the input signal, we are able to cancel the frequency component at $2 \omega_{0}$ that limits the performance of the lock-in amplifier as shown in Fig. 2.

Following on from Eqs. (2) to (6),

$$
v_{\Delta}(t)=V_{0} \sin \left(\omega_{0} t+\phi\right),
$$

$$
\begin{aligned}
v_{i \Delta}(t) & =V_{0} \sin \left(\omega_{0} t+\phi\right) \cos \left(\omega_{c} t\right) \\
& =\frac{V_{0}}{2}\left[\sin \left(\left(\omega_{0}+\omega_{c}\right) t+\phi\right)+\sin \left(\left(\omega_{0}-\omega_{c}\right) t+\phi\right)\right],
\end{aligned}
$$

$$
\begin{aligned}
v_{q \Delta}(t) & =V_{0} \sin \left(\omega_{0} t+\phi\right) \sin \left(\omega_{c} t\right) \\
& =\frac{V_{0}}{2}\left[\cos \left(\left(\omega_{0}-\omega_{c}\right) t+\phi\right)-\cos \left(\left(\omega_{0}+\omega_{c}\right) t+\phi\right)\right] .
\end{aligned}
$$

When $\omega_{c}=\omega_{0}$,

$$
\begin{aligned}
& v_{i \Delta}(t)=\frac{V_{0}}{2}\left[\sin \left(2 \omega_{0} t+\phi\right)+\sin \phi\right], \\
& v_{q \Delta}(t)=\frac{V_{0}}{2}\left[\cos \phi-\cos \left(2 \omega_{0} t+\phi\right)\right] .
\end{aligned}
$$
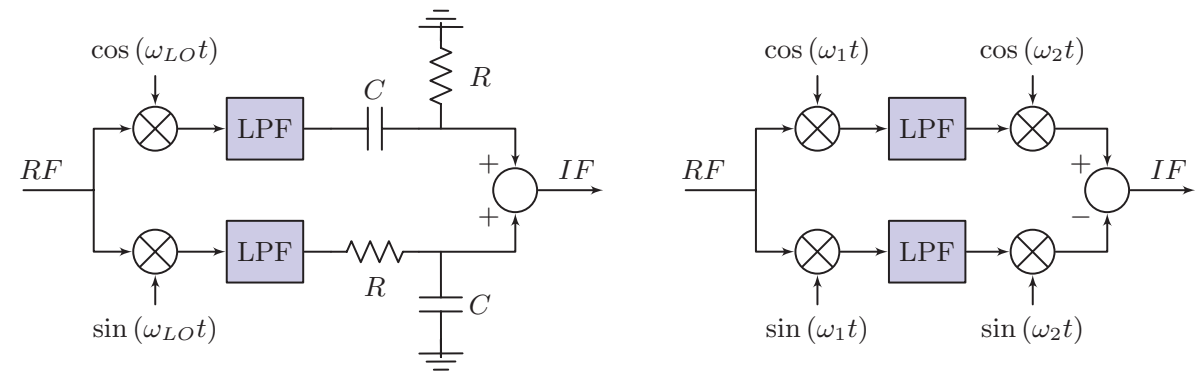

FIG. 4. The Hartley (left) and Weaver (right) architectures can perform image rejection in RF receivers. 


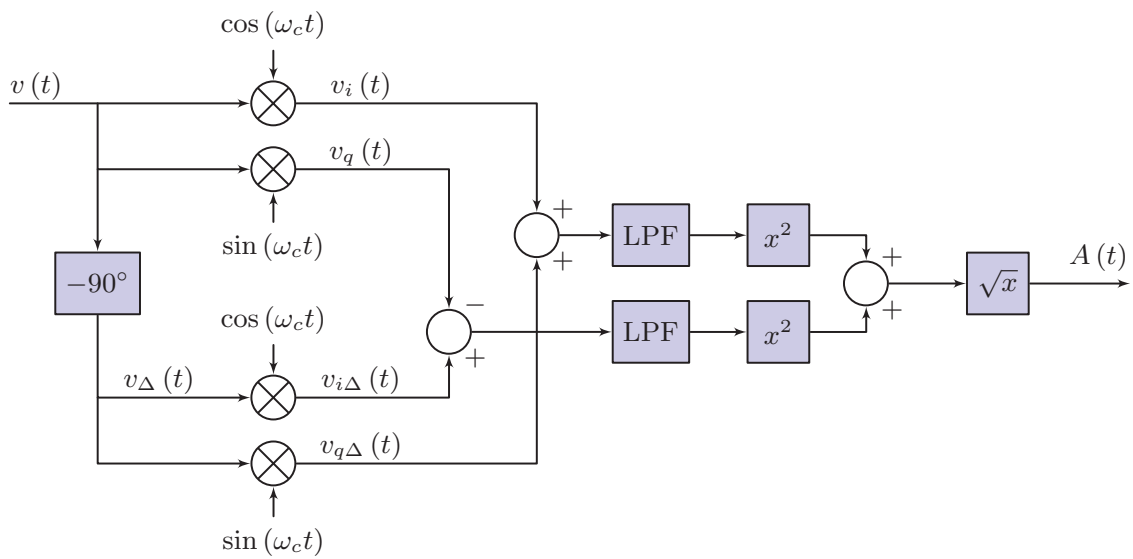

FIG. 5. The high-bandwidth lock-in amplifier amplitude estimation technique eliminates the frequency component at $2 \omega_{0}$. The bandwidth of the low-pass filters can be increased significantly compared to the standard technique.

Utilizing phase cancellation, the frequency component at $2 \omega_{0}$ can be mathematically canceled without filtering

$$
\begin{aligned}
v_{i}(t)+v_{q \Delta}(t)= & \frac{V_{0}}{2}\left[\cos \phi+\cos \left(2 \omega_{0} t+\phi\right)\right] \\
& +\frac{V_{0}}{2}\left[\cos \phi-\cos \left(2 \omega_{0} t+\phi\right)\right] \\
= & V_{0} \cos \phi, \\
v_{i \Delta}(t)-v_{q}(t)= & \frac{V_{0}}{2}\left[\sin \left(2 \omega_{0} t+\phi\right)+\sin \phi\right] \\
& -\frac{V_{0}}{2}\left[\sin \left(2 \omega_{0} t+\phi\right)-\sin \phi\right] \\
= & V_{0} \sin \phi .
\end{aligned}
$$

For a sinusoidal input, no low-pass filter is required to determine the amplitude

$$
\sqrt{\left(V_{0} \cos \phi\right)^{2}+\left(V_{0} \sin \phi\right)^{2}}=V_{0} .
$$

In practice, a low-pass filter is still required since circuit mismatches will prevent complete cancellation of the highfrequency oscillations. However, since the high-frequency oscillations have been reduced significantly, the low-pass filter
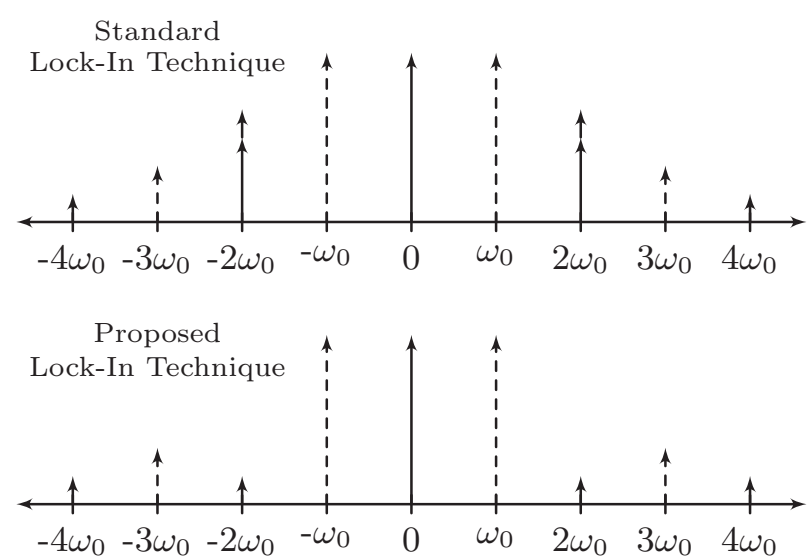

FIG. 6. Comparison of frequency spectra using the lock-in amplifier (top) and the proposed estimation technique (bottom) highlighting the input signal $(--)$ and the output signal prior to filtering $(-)$. does not need to be as aggressive and the measurement bandwidth can be increased considerably. In AM-AFM, the fundamental mode typically dominates the response of the microcantilever. Higher harmonics may be present, but these are typically not as significant as the fundamental mode and are situated far away in the frequency domain. Fig. 6 highlights that even with higher harmonics, the proposed technique is superior to the standard lock-in amplifier.

The achievable measurement bandwidth does depend on the input signal. Phase cancellation removes the components at $2 \omega_{0}$, potentially allowing a significant increase in the measurement bandwidth. Since the flexural modes of the microcantilever are generally well-spaced, high-bandwidth amplitude estimation is expected in the majority of AM-AFM applications. However, should undesirable frequency content be present in the vicinity of the resonance, the proposed amplitude estimation technique will perform no worse than the standard lock-in amplifier.

\section{H. Amplitude estimation in multifrequency AFM}

While the peak-hold technique ${ }^{11}$ may perform well in conventional AM-AFM, provided that higher harmonics are negligible, it is not suitable for multifrequency AFM experiments, such as bimodal excitation ${ }^{18-20}$ or advanced techniques, which rely upon feedback loops around higher modes. ${ }^{21,22}$ Based on the lock-in amplifier, the proposed technique is capable of accurately determining the amplitude of a specific mode in a multifrequency environment and is possibly the only high-performance technique currently available for such applications.

\section{SIMULATION}

Simulations were performed in MATLAB Simulink to validate the proposed amplitude estimation technique. In our experience with the Bruker DMASP microcantilever, the higher harmonic most likely to be most significant in the displacement signal in tapping mode AFM is the fourth harmonic, due to excitation of the second flexural mode. We therefore considered higher harmonics to demonstrate the 

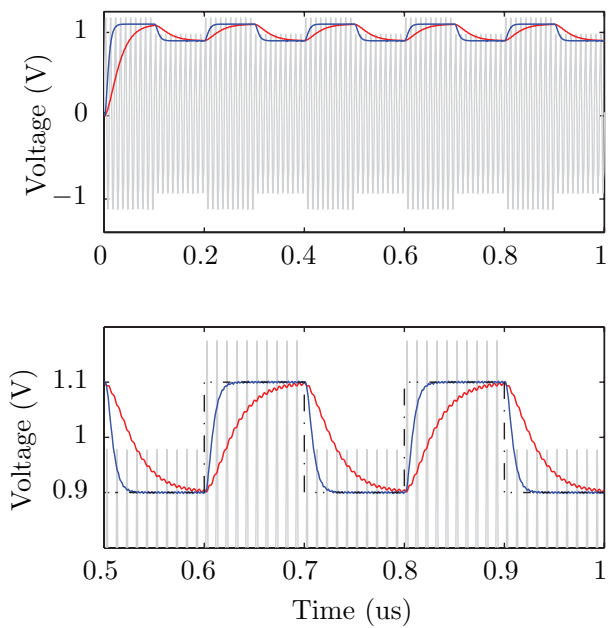

FIG. 7. Simulation results comparing the proposed amplitude estimation technique (LPF: $f_{c}=40 \mathrm{kHz}$ ) (blue dashed line) to the standard lock-in amplifier (LPF: $f_{c}=10 \mathrm{kHz}$ ) (red dashed line).

suitability of this technique in multifrequency AFM experiments. The fundamental component of the input signal was modulated with a square-wave with a period of $0.2 \mathrm{~ms}(5 \mathrm{kHz})$ to simulate changes in the surface topography of a sample

$$
v(t)=A(t) \cos \left(2 \pi f_{0} t\right)+0.1 \cos \left(4\left(2 \pi f_{0} t\right)+\phi\right)+n(t),
$$

where $A(t)=1+0.1 \operatorname{sgn}\left(\sin \left(2 \pi f_{a} t\right)\right)$, where $\operatorname{sgn}(x)$ is the signum function, $f_{a}=5 \mathrm{kHz}, f_{0}=100 \mathrm{kHz}, \phi=0.45 \mathrm{rad}$, and $n(t)$ is band-limited white noise.

Fig. 7 highlights the performance of the proposed scheme and the standard lock-in amplifier. Second order filters with bandwidths of $40 \mathrm{kHz}$ and $10 \mathrm{kHz}$ were utilized. The amplitude envelope depicted in Fig. 7 is that of the fundamental mode, not including the fourth harmonic. While RMS to DC conversion and the peak-hold technique cannot accurately determine the amplitude of the fundamental component, the lock-in amplifier is robust to the presence of other frequencies. The proposed technique clearly offers better performance and converges in two cycles, although convergence is dictated by the filter bandwidth and could be higher. With

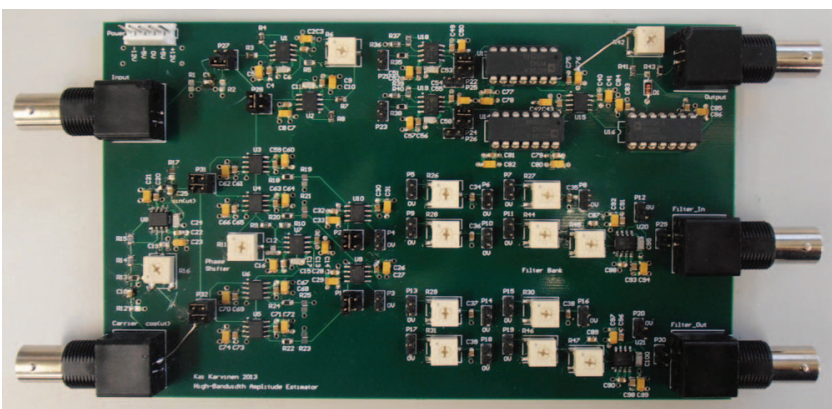

FIG. 8. High-bandwidth amplitude estimation circuit.

a low-pass filter bandwidth of $10 \mathrm{kHz}$, the output of the standard lock-in amplifier contains oscillation due to the second harmonic even with an aggressive filter and cannot accurately track the amplitude envelope. The presence of the fourth harmonic in the input signal does equate to the presence of the third and fifth harmonics in the output signal, however, these are more easily filtered and are not as significant as the second harmonic.

\section{IMPLEMENTATION}

An analog circuit, utilizing Analog Devices AD835 and AD734 four-quadrant multipliers, was designed and implemented for experimental verification. The AD835 is a highbandwidth multiplier with a full-scale range of $\pm 1 \mathrm{~V}$, a bandwidth of $250 \mathrm{MHz}$, and very low feedthrough. The AD734 is a high-accuracy multiplier featuring a full-scale range of $\pm 10 \mathrm{~V}$ with a $10 \mathrm{MHz}$ full power bandwidth capable of performing the analog square root operation. A host of high-performance amplifiers were utilized to enable operation in the megahertz regime, suitable for use with ultrahigh-frequency microcantilevers. Tunable all-pass filters were designed to adjust the phase delay of the input signal and to generate the quadraturephased signals required for multiplication. Selectable and reconfigurable filter stages were implemented to ensure tunability for individual applications. A custom printed circuit board was designed as shown in Fig. 8. Digital prototyping systems
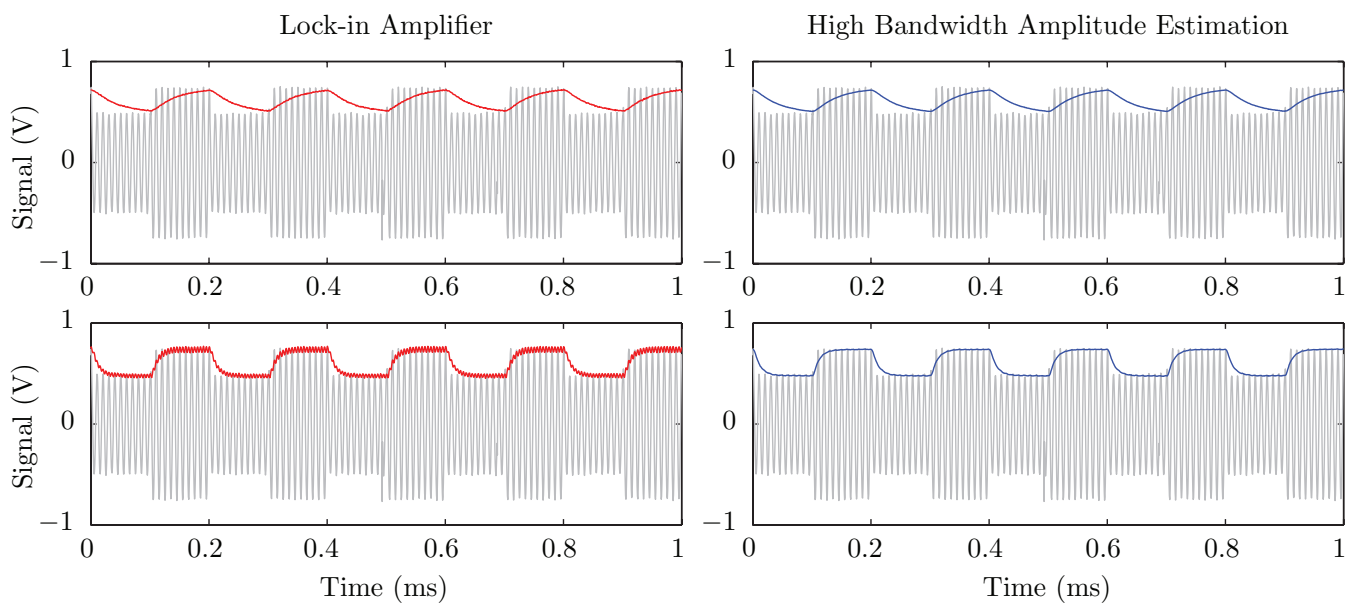

FIG. 9. Experimental results using series first-order low-pass filters with cutoff frequencies of $10 \mathrm{kHz}$ (top) and $40 \mathrm{kHz}$ (bottom). 


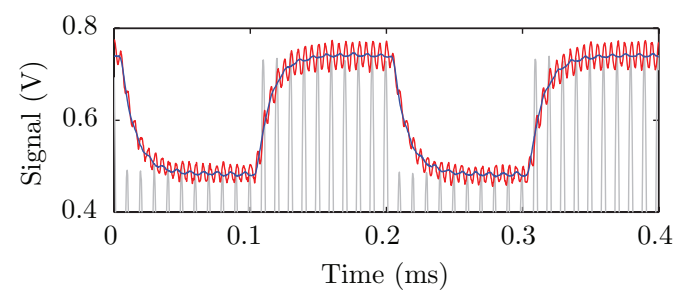

FIG. 10. Comparison of the lock-in amplifier (red dashed line) and highbandwidth technique (blue dashed line) with RC filters $f_{c}=40 \mathrm{kHz}$.

could be used to perform the square/square-root functionality in the baseband.

\section{EXPERIMENTAL RESULTS}

To demonstrate operation of the proposed technique, we applied a $5 \mathrm{kHz}$ square wave to the external modulation input of an Agilent 33120A function generator to produce an amplitude modulated $100 \mathrm{kHz}$ sinusoid similar to the signal used in simulation (Fig. 7).

The circuit could be configured as a standard lock-in amplifier and the high-bandwidth amplitude estimator, enabling a direct comparison between the two techniques.

The experimental results are highlighted in Fig. 9. There is clear agreement between the simulated and experimental results. To simplify implementation, first-order RC filter networks were connected in series to produce a second-order low-pass filter. The first-order filters had cutoff frequencies of $10 \mathrm{kHz}$ and $40 \mathrm{kHz}$. With the $10 \mathrm{kHz}$ filters, both techniques adequately eliminate the undesirable high-frequency components, however, the estimate cannot accurately track the profile of the signal. With the $40 \mathrm{kHz}$ filters, the high-bandwidth estimation technique is clearly superior.

The direct comparison of the techniques in Fig. 10 highlights a significant reduction in the high-frequency oscillations with the proposed amplitude estimation scheme. The small oscillations observed are due to a combination of feedthrough in the multiplier and DC offsets in the carrier. Such issues will limit the performance that can be obtained with this technique.

\section{CONCLUSIONS}

We have outlined a novel amplitude estimation technique, inspired by techniques employed in microwave and RF circuit design and modulated-demodulated control, which utilizes phase cancellation to significantly improve the performance of the lock-in amplifier. The scheme is simple to implement, capable of operating in a multifrequency environment and robust to high-frequency noise, all the while offering performance comparable to other high-bandwidth techniques. Experimental results verify the performance observed in simulation. We believe this technique could find application in both high-speed and multifrequency atomic force microscopy. It may also be relevant in other fields where a lock-in amplifier with improved measurement bandwidth is required.

${ }^{1}$ G. Binnig, C. F. Quate, and C. Gerber, Phys. Rev. Lett. 56, 930 (1986).

${ }^{2}$ Y. Martin, C. C. Williams, and H. K. Wickramasinghe, J. Appl. Phys. 61, 4723 (1987).

${ }^{3}$ Q. Zhong, D. Inniss, K. Kjoller, and V. B. Elings, Surf. Sci. 290, L688 (1993).

${ }^{4}$ T. Ando, Nanotechnology 23, 062001 (2012).

${ }^{5}$ T. R. Albrecht, P. Grütter, D. Horne, and D. Rugar, J. Appl. Phys. 69, 668 (1991).

${ }^{6}$ L. Chen, X. Yu, and D. Wang, Ultramicroscopy 107, 275 (2007).

${ }^{7}$ T. R. Rodríguez and R. García, Appl. Phys. Lett. 82, 4821 (2003).

${ }^{8}$ N. Kodera, D. Yamamoto, R. Ishikawa, and T. Ando, Nature (London) 468, 72 (2010).

${ }^{9}$ M. Shibata, H. Yamashita, T. Uchihashi, H. Kandori, and T. Ando, Nat. Nanotechnol. 5, 208 (2010).

${ }^{10}$ T. Uchihashi, R. Iino, T. Ando, and H. Noji, Science 333, 755 (2011).

${ }^{11}$ T. Ando, N. Kodera, E. Takai, D. Maruyama, K. Saito, and A. Toda, Proc. Natl. Acad. Sci. U.S.A. 98, 12468 (2001).

${ }^{12}$ J. Kokavecz, Z. Tóth, Z. L. Horváth, P. Heszler, and Á. Mechler, Nanotechnology 17, S173 (2006).

${ }^{13}$ C. Kitchin and L. Counts, RMS to DC Conversion Application Guide, 2nd ed. (Analog Devices, Inc., 1986).

${ }^{14}$ T. Ando, T. Uchihashi, and T. Fukuma, Prog. Surf. Sci. 83, 337 (2008).

${ }^{15}$ B. Razavi, in Proceedings of the Custom Integrated Circuits Conference, 1998 (IEEE, 1998), pp. 393-400.

${ }^{16}$ K. Lau, D. E. Quevedo, B. J. G. Vautier, G. C. Goodwin, and S. O. R. Moheimani, Control Eng. Pract. 15, 377 (2007).

${ }^{17}$ K. S. Karvinen and S. O. R. Moheimani, "Modulated-demodulated control: Q control of an AFM microcantilever," Mechatronics (in press).

${ }^{18}$ R. Garcia and E. T. Herruzo, Nat. Nanotechnol. 7, 217-226 (2012).

${ }^{19}$ R. Proksch, Appl. Phys. Lett. 89, 113121 (2006).

${ }^{20}$ J. R. Lozano and R. Garcia, Phys. Rev. Lett. 100, 076102 (2008).

${ }^{21}$ S. D. Solares and G. Chawla, J. Appl. Phys. 108, 054901 (2010).

${ }^{22}$ D. Ebeling and S. D. Solares, Beilstein J. Nanotechnol. 4, 198 (2013). 